\title{
Estudio de 258 Prematuros en el Primer Año de Vida
}

\author{
Extracto y comentario del artículo publicado en \\ Revista chilena de Pediatría 1941; 12(1): 1 -31 y 93 -151
}

OSCAR ILLANES BENÍTEZ ${ }^{1}$ y RENÉ ALFARO DE LA CERDA ${ }^{1}$

Comentario: LUISA SCHONHAUT B. ${ }^{2}$ y MARCELA PÉREZ R. ${ }^{2}$

1. Casa Nacional del Niño, Clínica Extraordinaria de Pediatría, Profesor A. Ariztía.

2. Departamento de Pediatría, Facultad de Medicina Clínica Alemana - Universidad del Desarrollo.

Key words: Pediatric history, preterm infant.

Palabras clave: Prematuros, historia pediatría.

Agradecimientos: A los doctores Clara Román y Jorge Torres Pereyra por desafiar los paradigmas de los cuidados neonatales y regalarnos sus vivencias, anécdotas y experiencias, que de alguna manera esperamos se reflejen en el presente artículo. Agradecemos también la generosidad del Sr. Carlos Sánchez, quien compartió con nosotras un pedazo de la historia de los niños del país, plasmado en las memorias de la Casa Nacional del Niño, institución destacada en el cuidado del niño abandonado, que está vigente hasta el día de hoy.

\section{Introducción}

A lo largo de los últimos casi 80 años, nuestra revista ha trasmitido el testimonio de grandes progresos en el cuidado de los niños. Quizás los más sorprendentes han ocurrido en la medicina de alta complejidad, en la atención de los niños gravemente enfermos y en los cuidados neonatales, especialmente de prematuros cada vez más pequeños ${ }^{1,2}$.

En los años 30, la sobrevida de un recién nacido de menos de 1000 gr era prácticamente anecdótica. De acuerdo con la definición de Yilpoo, se consideraba prematuro a "todo niño nacido con peso inferior a 2500 gramos, sin tener en cuenta la duración del embarazo ni los signos de parto prematuro". Esta definición, predominantemente clínica, se fundamentaba en la observación que, independiente de la causa de bajo peso de nacimiento, estos niños "requieren un trato especial, revelando, al mismo tiempo, características particulares, que se traducen, de preferencia por dificultades en su crianza".

Sin embargo, esta definición ya comenzaba a generar controversias y no se lograba uniformidad de criterio respecto a aspectos que actualmente parecen tan básicos. A diferencia de lo planteado por Yilpoo, los norteamericanos consideraban como punto de corte las cinco libras (equivalente a 2268 gr) y en la mayoría

Trabajo recibido el 10 de junio de 2010.

Correspondencia a:

Luisa Schonhaut B.

E-mail: Ischonhaut@alemana.cl 
de las maternidades de Santiago utilizaban el "criterio obstétrico" de 2700 gr, ya que consideraban que este "sería el límite mínimo compatible con una gestación de nueve meses". Otros autores incorporaban además la edad gestacional y longitud al nacimiento. Basado en estos principios, la Liga de las Naciones a su vez sub-clasificaba a los prematuros en previables o viables (figura 1). Si bien el límite de sobrevida propuesto para estos últimos era de $1000 \mathrm{gr}$, en la práctica, en nuestro país, las acciones terapéuticas iban dirigidas a prematuros de pesos algo superiores (1 200 a 1500 gr) criterio que sentenciaba la opción de sobrevida.

Otros autores, como Rohmer, referían que el pronóstico guardaba relación con los factores que originaron la prematurez, por ende, proponían clasificar a los prematuros en 3 grupos:

“1) Los sanos, hijos de padres sanos, en que el embarazo ha sido interrumpido precozmente por una causa fortuita.

2) Los con signos de debilidad congénita, cuyo desarrollo ha sido retardado por una enfermedad de la madre, o que ellos mismos son enfermos; estos niños pueden ser nacidos de término, pero lo más a menudo la causa de la debilidad ha sido motivo de la interrupción prematura del embarazo.

3) Aquellos nacidos de término y con las características de la madurez, pero en los cuales el peso y la estatura son inferiores a lo normal, siendo su conducta posterior igual al de los niños normales (hipoplasia, embarazo gemelar, etc)."

"El niño hipoplásico sería un niño dañado en su plasma germinal o en la vía intrauterina, lo que hace de él siempre un individuo funcionalmente inferior (Ariztia) ${ }^{3}$."

Probablemente al segundo y tercer grupo de Rohmer pertenecía la mayoría de los prematuros, que sorteando las dificultades de los primeros días de vida, llegaban al Asilo de la Casa Nacional del Niño, sobre todo por razones sociales, pero también por motivos médicos, como la necesidad de aislamiento de una madre bacilífera, o por indicaciones higiénicas y morales. "Las causas que han motivado el ingreso de estos niños son muy variadas y corresponden a las finalidades que, en general, cumple el establecimiento, consistentes en asistir o asilar transitoriamente, mientras sea indispensable, aquellos niños cuyas familias atraviesan situaciones aflictivas de naturaleza económica, higiénica o moral; y en asistir al huérfano y al abandonado, a fin de obtener de ellos, en la edad adulta, individuos capaces de desenvolverse en forma independiente y eficaz (Illanes \& Alfaro 1941)."

La Casa Nacional del Niño, fundada el año 1751 como Casa de Expósitos, fue pionera en la protección de la infancia probablemente a nivel latinoamericano. En un comienzo, en su orientación de beneficencia, ofrecía un cuidado básico a los niños que estaban en situación de abandono; no obstante, motivados por las dramáticas condiciones de salud y elevadas tasas de mortalidad de los "asilados", comenzó a involucrarse cada vez más el cuidado pediátrico de los niños, dando origen a una de las primeras cátedras de Pediatría del país ${ }^{4}$, con una orientación docente-asistencial y de investigación en infancia.

En un extenso artículo, del que reproducimos textualmente sólo las conclusiones, Oscar Illanes y René Alfaro relatan el seguimiento de 258 prematuros admitidos en la Casa Nacional del Niño, entre los años 1930 y 1939.

Para elaborar el comentario, recorrimos los artículos relacionados con la mortalidad y evo-

Figura 1. Clasificación de prematuros de acuerdo a la Liga de las Naciones (1930)

\begin{tabular}{|llll|}
\hline & Edad gestacional & Peso de nacimiento & Longitud \\
\hline Recién nacido de término & 38 semanas o más de vida intrauterina & más de $2500 \mathrm{~g}$ & Más de $47,1 \mathrm{~cm}$ \\
\hline Prematuro viable & 29 hasta el final de 37 semanas & 1000 a $2499 \mathrm{~g}$ & 35,1 a $47 \mathrm{~cm}$ \\
\hline Prematuro previable & 22 hasta el final de 28 semanas & 400 a $999 \mathrm{~g}$ & 28 a $35 \mathrm{~cm}$ \\
\hline
\end{tabular}


lución de los prematuros, publicados en nuestra revista desde aquellos años. Transcribimos algunos párrafos en forma textual de modo de ilustrar el paradigma vertical y determinista que predominaba en la atención de los niños.

\section{Artículo original}

La propia naturaleza de este trabajo, cuya finalidad es mostrar algunas particularidades generales del material de prematuros de la Casa Nacional del Niño, sobre su etiología, desarrollo, morbilidad, mortalidad, etc, constituye una exposición de carácter analítico. Como se comprende sería materialmente imposible resumir, en breves frases dicha exposición, si tratáramos de abarcar todos los aspectos dignos de mencionarse.

Por consiguiente, este último capítulo tiene, más bien por fin llamar la atención hacia aquellos puntos que, a nuestro juicio deben ser examinados con un mayor detenimiento que el que depara la rápida lectura de conclusiones. Esto, sin perjuicio naturalmente de ir proporcionando, de paso, los antecedentes indispensables que permitan al lector formarse un concepto respecto a los temas tratados, si bien muy general.

1) El material de estudio estuvo formado por 258 observaciones de lactantes prematuros, de acuerdo con el concepto pediátrico de Arvo Yilppo, cuyo ingreso se ha verificado para dar solución a problemas de orden médico social de la colectividad.

Gran número de ellos mostró al ingreso, además de las manifestaciones propias a la prematurez, comunes a todos, las diversas fases de la desnutrición cualitativa y cuantitativa, no siendo raros los casos de descomposición, y afectos de procesos infecciosos o de otra índole.

Como a todos los demás niños, se les practicó a su llegada un examen con el fin de pesquisar las enfermedades infecto-contagiosas para los fines epidemiológicos consiguientes y conocer su estado nutritivo.

A continuación, fueron aislados en una sala especial que se diferencia de las otras en que tiene su ambiente temperado a $22-25^{\circ}$ y con un buen grado higrométrico.
Cuando estos niños han presentado falta de su regulación térmica, se les ha colocado botellas calientes. La alimentación ha sido de leche humana exclusiva en las primeras semanas, dado, al pecho, como se hacía en años atrás, o, como se hace ahora, por cucharaditas. La sonda por vía nasal, utilizada en los casos de incompleto desarrollo del reflejo de la deglución, no ha presentado los peligros descritos para este procedimiento. Se atribuye este éxito, como los otros que se relacionan con el cuidado del prematuro, a la idoneidad del personal de enfermeras.

2) Las principales causas de prematurez en el material de la tesis ha sido suministrado por el grupo de los factores denominados evitables, es decir, las enfermedades de la madre: la tuberculosis, $31,3 \%$, la sífilis, $8 \%$; las enfermedades consuntivas $5,5 \%$. El factor más importante de las causas no susceptibles de corrección, los partos múltiples, figura con un 10,1\%.

Las condiciones higiénicas del país nos permiten suponer que nuestra estadística refleja, si no con exactitud, por lo menos en forma aproximada, la frecuencia con que dichos factores intervienen en la producción de la prematura en nuestro medio.

La revisión de las estadísticas en nuestras Maternidades debe suministrar resultados más concordantes con la realidad sobre este importante aspecto del estudio del niño prematuro, trabajos que hasta la fecha no han sido realizados.

3) La cuarta parte de los prematuros presentó manifestaciones de debilidad congénita, cuya mortalidad, $32,6 \%$ fué bastante mayor que la del grupo de los prematuros sin debilidad congénita.

4) El síndrome asfíctico-convulsivo. $\mathrm{Su}$ denominación nos ahorra explicaciones sobre su naturaleza. Es preciso hacer notar que en la literatura extranjera se describe el cuadro asfíctico sin ir acompañado de convulsiones. Dicho síndrome, estudiado en el primer trimestre de la vida, se presentó, de modo preferente, en los niños con un peso de nacimiento menor a dos mil gramos. Lo mortalidad fué $75 \%$. Las lesiones que con más frecuencia se encontraron en la autopsia fueron las de bronconeumonías, a continuación las hemorragias cerebra- 
les. También se observó hipertrofia de timo y tuberculosis del primer trimestre, causas que no mencionan los autores extranjeros. En los niños que sobrevivieron, el síndrome se manifestó concomitantemente a procesos gripales o bien se ignoró su etiología.

5) En un 34\% los prematuros tuvieron regurgitaciones; la cuarta parte de éstos fueron vomitadores además. Tampoco hemos encontrado referencias sobre estas perturbaciones en el niño prematuro en la literatura extranjera. En general, las regurgitaciones, acompañadas o no de vómitos, se presentaron de manera continua o intermitente, durante las primeras semanas o los primeros meses de la vida. $\mathrm{Su}$ comienzo se relaciona con frecuencia a los aumentos en la cantidad de leche ingerida; en cuanto a su término no parece tener nexo con alguna condición manifiesta, propia del niño o del ambiente, operándose en forma paulatina y espontánea. Estas observaciones clínicas, como la experiencia terapéutica, permiten confirmar la opinión de algunos autores que los vómitos y las regurgitaciones tienen su origen en una reducida capacidad del estómago del prematuro para el alimento suministrado, a lo que se agrega una perturbación funcional vegetativa de la motilidad gástrica, atonía en las regurgitaciones, hipertonía en los niños vomitadores. Por lo último, es comprensible que las formas más intensas y pertinaces de estos trastornos se presenten en aquellos niños con diástasis exudativa neuropática.

6) El raquitismo: Se presentó en 104 niños o sea, en el $80 \%$. En la mayoría de los casos se inició en el tercer mes y su regresión ocurrió en las postrimerías del primer año. $\mathrm{La}$ evolución tiene relación con la eficacia de los métodos de suministro de la vitamina $\mathrm{D}$. Se vió esta evolución en 74 niños que recibieron tratamiento curativo con la vitamina dada en dosis fraccionada durante un tiempo prolongado; según los métodos preconizados hasta hace poco. También se observó en otros 20 niños, no obstante haberse instituido un tratamiento profiláctico. Por fin, en otros diez niños que completan el total de 104; fueron comprendidos en el material de experimentación del trabajo de Schwarzenberg y Cousiño, sobre la eficacia de la terapia curativa mediante el golpe vitamínico. Estos niños se dejaron deliberadamente sin protección anti-raquítica y una vez que presentaron raquitismo florido, se les aplicó el golpe. La evolución fué en estos casos considerablemente más breve que en los tratados conforme a los otros métodos.

El 20\% restante, 26 prematuros se vieron libres de raquitismo durante los doce meses en que se observaron. Diez de ellos siguieron tratamiento profiláctico, de acuerdo con los procedimientos antiguos. En la mayoría de estos casos, la administración comenzó entre los tres y los cuatro y medio meses. No podría afirmarse que la no aparición del cuadro raquítico se debió de manera exclusiva a la oportunidad del tratamiento y a las cantidades de vitamina que se suministraron. En efecto, experiencias de autores extranjeros revelan que la protección no se asegura en todos los casos, ni siquiera a partir del primer mes de la vida, con dosis diarias más altas que las nuestras durante largos meses. En dichos diez casos han intervenido, probablemente factores individuales, como un menor requerimiento vitamínico de protección o un más adecuado aprovechamiento del principio anti-raquítico. Finalmente, en otros 16 prematuros, con los cuales se completa el $20 \%$ libre del proceso carencial, no hubo tratamiento alguno, o bien éste fué ínfimo. Aquí cabe la explicación de una tendencia natural a no hacer el cuadro, por la influencia de los factores individuales enunciados.

7) El cuadro hemático evolucionó en la forma clásica descrita. Hubo tendencia a la poliglobulia y a la hipercromemia en el primer mes y al descenso a valores subnormales en les meses siguientes, en especial en el tercero. Fué también frecuente el valor globular menor que uno, es decir, la hipocromemia, como en la clorosis.

Los tratamientos preconizados desde tiempo atrás: administración de hierro, cobre $\mathrm{y}$ manganeso, de preparados de hígado, etc, no dieron resultados decisivos; experiencia que concuerda con lo observado por otros autores. Conviene hacer notar que en la Casa Nacional del Niño ha dejado de constituir la anemia del prematuro un problema terapéutico. Gracias a las transfusiones de sangre se ha logrado corregirla en el curso de pocos días o semanas, 
en forma duradera. La transfusión de sangre ejerce una modificación tan intensa que, con frecuencia, es dable observar un alza de la tasa de glóbulos rojos y del índice hemoglobínico a límite superiores a los seis millones y al 100\%, respectivamente.

8) El antecedente de tuberculosis materna incidió en la elevada frecuencia del 40,2\%. Esta proporción, además de traducir la suma difusión de este mal, refleja también la preferencia que se da en el establecimiento al ingreso de los hijos de tuberculosa, para salvarlos del contagio. La mortalidad en los prematuros pertenecientes a este grupo etiológico de la prematurez fué de $45,2 \%$, mayor que la ofrecida por todo el material, $41,4 \%$.

Importa recalcar el siguiente hecho de importancia higiénica y social: las madres de casi la mitad de estos niños murieron, en su gran mayoría, en los días o en las semanas siguientes al parto.

Se confirma que la posibilidad de contaminación está en relación directa con la duración del contacto. El 10\% de los separados al nacer, enferman de tuberculosis y el $33 \%$ de los que permanecieron en contacto enfermaron. Todos los prematuros tuberculosos, que fueron doce, murieron: diez antes del término del segundo semestre, y 2, durante el tercer trimestre.

9) La lúes materna, aislada o asociada a otra causa de prematurez, se mostró en el 13,9\% (33 niños). De los 33 murieron 17, es decir, el $51,5 \%$, que es la mortalidad más alta con respecto a un factor etiológico de prematurez determinado; siete por Lúes y los otros diez, por afecciones intercurrentes. De los 16 sobrevivientes, 3 hicieron lúes y curaron después de recibir el tratamiento correspondiente.

10) La mortalidad ascendió al $41,4 \%$. Muy semejante a la de algunos países mejor organizados socialmente que el nuestro. Estuvo en relación directa con la disminución del peso de nacimiento. Considerada con respecto a la edad fué mínima en el período de recién nacido y a partir de aquí se elevó con rapidez hasta alcanzar en el cuarto mes la cima con un $24,2 \%$; en el quinto bajó a $12,1 \%$ y en el resto del año descendió a proporciones mínimas. Esta curva es algo diversa a las que se da en los textos, en que el nivel más alto está precisamente en la época de recién nacido, baja muy rápidamente hasta el cuarto mes, en que vuelve a alzarse, pero en forma discreta haciéndose muy baja en el resto del año. La diferencia principal radica, como se ve, en el primer mes. Esto se explica, a nuestro juicio porque sólo ingresan aquellos prematuros que se han librado de los accidentes propios del parto y los que presentan una vitalidad compatible con las contingencias del cuidado deficiente previo al ingreso. A lo anterior se agrega el cuidado correcto que se les prodiga en el establecimiento.

El aumento de la mortalidad señalado en el cuarto mes que describen también algunos autores, como Sales y Valléry, Radot y Feer, si bien no en forma tan acentuada como en nuestra curva se explica según nuestro entender, por el ambiente del establecimiento cerrado del Asilo favorable al desarrollo y difusión de las infecciones, en especial las gripales, a lo que se agrega la pronta aparición de la anemia y del raquitismo, que exacerban la disergia.

Se hace notar, sin embargo, que se ha hecho evidente un progreso marcado en el cuidado de estos niños, pues por el estudio anual de la mortalidad se ve un franco descenso de ella, que en los últimos años tiende a fluctuar entre 20 y $30 \%$.

Los procesos infecciosos intercurrentes especialmente las: bronconeumonías, las sepsis y las otitis, constituyeron el grupo causal de muerte más numeroso: $66,3 \%$. A continuación las infecciones connatales (tuberculosis y lúes): $14 \%$; los factores de mortalidad precoz (debilidad congénita, hemorragias cerebral y umbilical): $7,4 \%$; los trastornos nutritivos: $6,5 \%$ y otras causas: $5,6 \%$.

Hemos explicado el motivo de la mayor frecuencia de los procesos infecciosos en las causas de muerte y por qué intervienen en un plano secundario los factores de mortalidad precoz. La reducida frecuencia de los trastornos nutritivos se debe a la naturaleza asistencial del establecimiento.

En el medio social circundante deben ser naturalmente, más comunes los fallecimientos por las causas precoces y por los trastornos nutritivos y menos frecuentes los consecutivos a procesos infecciosos, que en el material de estudio. 
El estudio de las estadísticas de nuestras Maternidades debe suministrar valiosos datos sobre estos particulares.

11) En los dos o tres primeros meses, la alimentación fue exclusivamente la leche humana. Se comprobó que los progresos ponderales más altos se observaron con cocientes energéticos también elevados, en especial, en los prematuros de menor peso. Con todo, esta conclusión de alcance general debe estar supeditada a las características del desarrollo pondoestatural de cada niño, pues los hay que exigen un mayor requerimiento calórico y otros uno menor, para realizar una gráfica ponderal progresivamente ascendente.

En los prematuros que mostraron una gráfica ponderal estacionaria, a pesar de estar sometidos a la alimentación natural con un cociente energético elevado, se les hizo a su dieta agregados de proteínas, en forma de leche albuminosa o de otros preparados análogos. También de babeurre adicionado de cocimiento de mantequilla y harina. Fue buena la tolerancia a estas modificaciones y satisfactorio fué el progreso ponderal que se perseguía.

12) Con mucho éxito se utilizaron las transfusiones sanguíneas en aquellos prematuros en que convenía la indicación de una terapéutica de estímulo; en especial, los que presentaron a su ingreso un estado nutritivo muy precario, manifestando un escaso potencial vital y aquellos que experimentaron una desmejoría del estado general por causa de procesos infecciosos intercurrentes o a consecuencia de trastornos nutritivos, sean éstos por intolerancia alimenticia o debidos a estados infecciosos.

Con las transfusiones se obtuvieron, secundariamente, las modificaciones del cuadro sanguíneo ya señaladas.

Ha tenido también resultados favorables el uso del Prolán, a que se ha recurrido en las primeras semanas de la vida, a fin de estimular el desarrollo de algunos prematuros. Además de entonar la curva del peso, se puede observar con este hormón una mejoría del apetito y del estado psíquico que se manifiesta por mayor vivacidad.

13) Los nacidos con más de dos mil gramos no alcanzaron, en su mayoría, a triplicar el peso antes de cumplir el año de edad, lo que consiguieron los nacidos con un peso menor. El crecimiento de la talla tuvo un promedio de progreso anual muy semejante en todos los niños, $20 \mathrm{cms}$ más o menos. No se dispuso de los datos pertinentes de los niños no prematuros del Asilo, a fin de hacer comparaciones susceptibles de reflejar en este sentido, características propias del prematuro.

\section{Comentario}

En los años en que se escribió el artículo, Chile concentraba una de las más altas tasas de Mortalidad Infantil del mundo. La gran mayoría de los partos eran domiciliarios, los registros estadísticos carecían de una representatividad nacional En el año 1939, el entonces Ministro de Salubridad Salvador Allende describía que de cada 20 partos uno era mortinato y de cada 100 nacidos vivos, la mitad moría en las primeras horas, 10 durante el primer mes y 25 antes de alcanzar el primer año de vida ${ }^{5,6}$.

En la Casa Nacional del Niño, cerca del $40 \%$ de los prematuros que ingresaban fallecían anualmente. Los autores destacaban que la mayor mortalidad del "material de estudio" se concentraba en el cuarto mes y se debía principalmente a enfermedades infectocontagiosas, a diferencia de lo reportado en la literatura científica: "Esto se explica, a nuestro juicio porque sólo ingresan aquellos prematuros que han librado de los accidentes propios del parto y los que presentan una vitalidad compatible con las contingencias del cuidado deficiente previo al ingreso. A lo anterior se agrega el cuidado correcto que se les prodiga en el establecimiento."

Entre las afecciones que aquejaban a los prematuros incluidos en el estudio destacaba la "desnutrición cualitativa y cuantitativa, no siendo raros los casos de descomposición, y afectos de procesos infecciosos o de otra indole", la "debilidad congénita", el "síndrome asfictico convulsivo" y el raquitismo.

No obstante las condiciones particulares de estos niños, los autores intuyen que sus observaciones son extrapolables a la realidad del país y destacan la falta de publicaciones sobre el tema, instando a las maternidades a revisar 
las estadísticas de modo de "suministrar resultados más concordantes con la realidad sobre este importante aspecto del estudio del niño prematuro".

Como respuesta a dicho desafió, Kohan analizó las estadísticas de la Maternidad del Hospital Barros Luco, durante los años 1938 y 1939 , destacando que $12,2 \%$ de los recién nacidos eran considerados prematuros de acuerdo al criterios pediátrico, $(<2500 \mathrm{gr})$, tasa que aumentaba a $26,6 \%$ si se considera además la edad gestacional. Sólo un prematuro fue "previable"7.

"Como detalles agregados, anotemos que el mayor número de hijos lo ha tenido una señora de 34 años. Tenía los siguientes antecedentes: 15 hijos. 2 abortos espontáneos, 4 hijos vivos. Reacción de Wassermann (-). Da a luz un niño de 1,100 gr., muerto en el útero. Fijémonos en la gran cantidad de hijos que ha tenido esta mujer, pero observemos que sólo 4 de estos niños están vivos, fenómeno muy común entre las mujeres del pueblo chileno.”(Kohan 1941).

En su estudio Kohan concluye que el "problema de la prematuridad es más obstétrico que pediátrico", siendo atribuible más a factores sociales que a factores biomédicos; en aquellos años las principales causas reconocidas de prematuridad eran la edad avanzada de la madre, los partos gemelares, la ilegitimidad, la sífilis y la "deficiente nutrición de la madre de clase baja".

Similares fueron las conclusiones de Illanes y Alfaro, quienes describieron que más de la mitad de los niños tenía antecedentes de "factores denominados evitables, es decir, las enfermedades de la madre" como TBC, sífilis materna o "enfermedades consuntivas", y $10 \%$ correspondía a causas no evitables como son los partos múltiples.

Paralelamente Eggers hizo un seguimiento a más largo plazo de los niños nacidos prematuros, destacando la importancia del entorno en el desarrollo infantil. En su estudio demostró que “...los prematuros con déficit mental provienen en gran parte de familias a su vez taradas, sin considerar además al factor trauma obstétrico, cuyo rol por el desempeñando aun no está lo suficientemente esclarecido en sus significados etiológicos, como causa de atraso mental. Los que sobreviven el tercer año quedan incorporados en su mayor parte a las actividades y destino de su generación." Entre las secuelas de la prematuridad Eggers describió los cuadros de hipo e hipertonía, hidrocefalia secundaria e "idiocía"8.

En la década de los 50 se creó el primer Centro de Prematuros Latinoamericano, en el Hospital Luis Calvo Mackenna, con gran impacto en la mortalidad infantil tardía y en la calidad de sobrevida de los pequeños ${ }^{9,10}$. Esta iniciativa se fue replicando en otros lugares de Santiago y en regiones, desarrollándose importante actividad científica a nivel nacional.

La realidad que nos muestran los distintos estudios son similares hasta los años 70; los recursos eran pobres, las diferencias en las estadísticas eran explicables principalmente por el tiempo de seguimiento, edad de ingreso y los factores médico sociales específicos: los partos en un porcentaje importante carecían de atención profesional; las enfermedades como tuberculosis, sífilis, pobreza y desnutrición eran flagelos importantes que iban en vías de la erradicación, los menores de 1000 g morían casi sin opción. Predominaba la mortalidad neonatal precoz. El pronóstico y desarrollo de los sobrevivientes dependía fundamentalmente de factores ambientales ${ }^{11}$.

En los últimos 40 años, gracias al esfuerzo pediátrico y al progreso obstétrico en la atención prenatal, durante el parto y el puerperio, mejoró significativamente la sobrevida y pronóstico de los prematuros.

Algunos de los hitos destacables, que cambiaron la historia de los prematuros fueron, la administración de corticoides en presencia de amenaza de parto prematuro en los años 60; el descubrimiento de los beneficios de la presión positiva para insuflar los pulmones, a través del CPAP nasal; la implementación de unidades de cuidados intensivos neonatales y ventilación mecánica, que se expandirían recién en los ' $70-80^{12}$; luego vino el uso de Surfactante, que en Chile fue administrado en forma universal a partir del año 2000.

El año 1998 se formó una Comisión Nacional que dio origen al Programa de Seguimiento de Prematuros, con el propósito de promover 
la equidad y calidad en el cuidado de prematuros extremos, mediante la implementación tecnológica y profesional de las unidades neonatales, la capacitación, el desarrollo de guías clínicas, que se han ido incorporando como Garantías Explícitas en Salud (GES) y la suplementación nutricional ${ }^{13}$.

Gracias a las estrategias mencionadas, se ha mejorado sustancialmente el pronóstico de este grupo de recién nacidos de gran vulnerabilidad, brindándonos la oportunidad de maravillarnos con los logros de prematuros de menos de $500 \mathrm{~g}$ o 25 semanas de gestación. ${ }^{14}$ Ellos nos plantean nuevos e impensables desafíos, tanto profesionales como éticos, de los cuales serán testigos las próximas generaciones de pediatras.

\section{Referencias}

1.- Salas R, Sanhueza L, Maggi L: Factores de riesgo y seguimiento clínico en prematuros menores de 1000 gramos. Rev Chil Pediatr 2006; 77 (6): 577-88.

2.- Llanos A y Grupo Hospital Sótero del Río: Morbilidad y mortalidad del RN menor de 1500 g: Experiencia de un hospital regional de la red internacional Vermont Oxford. Rev Chil Pediatr 2006; 77 (4): 363-74.

3.- Ariztía A: Los conceptos sobre la constitución individual y la fisiopatología del lactante. Ensayo de aplicación clínica. Librería Nacimiento. Santiago. 1933.

4.- Ariztía A: Sobre Orientaciones Actuales De La Enseñanza de la Pediatría. (Lección Inaugural Del Curso Extraordinario De Pediatría). Rev Chil Pediatr 1934; 5: 169-86.

5.- Allende $S$ : La realidad médico-social chilena: (síntesis)/
Salvador Allende G. [Santiago]: Minist. de Salubridad, Previsión y Asistencia Social, 1939 ([Santiago]: Lathrop) $216 \mathrm{p}$.

6.- Schonhaut L: La Mortalidad Infantil en Chile estudiada por la Sociedad de las Naciones. Rev Chil Pediatr 2007; 78 (2): 202-10.

7.- Kohan R: Algo más sobre prematuridad. Rev Chil Pediatr 1940; 11 (8): 596-607.

8.- Eggers F: El prematuro y algunas características especiales de su patología. Rev Chil Pediatr 1940; 11 (8): 585-95.

9.- Howard J, Ebensperger I, Román C, O livos P, Cofre P, Soto R: Evolucion extra hospitalaria en los primeros 12 meses de vida de los prematuros ingresados al centro de prematuros del Hosp. L. Calvo Mackenna. 1956-1957. Rev Chil Pediatr 1959; 30 (3).

10.- Agüero G, Moreno L, Roman C, Ebensperger I, Howard $J$, Olivos P: Causas de muerte del niño prematuro en la primera semana de vida. Centro de Prematuros: Hospital "Luis Calvo Mackenna". Rev Chil Pediatr 1960; 31 (2): 55-64.

11.- Beca JP, Román C, Zubaty LY, Abourbih J: Estudio de Desarrollo de Niños Considerados de Alto Riesgo Neurológico en el Período Neonatal. Rev Chil Pediatr 1980; 51 (2): 97-104.

12.- Weldt E, Hering E, Valenzuela B, Angulo GY, Neira A: Seguimiento de Ninos con Peso de Nacimiento Inferior A 1.500 g. Rev Chil Pediatr 1986; 57(1): 51-6.

13.- Morgues $M$, Henríquez $M$, Tohá $D$, et al: Comisión Nacional de Seguimiento del Prematuro. MINSAL. Sobrevida del Niño Menor de 1500 Gramos en Chile. Rev Chil Obstet Ginecol 2002; 67: 100-5.

14.- Hubner M, Nazer J, Juárez de León G: Estrategias para Mejorar la Sobrevida del Prematuro Extremo. Rev Chil Pediatr 2009; 80 (6): 551-9. 